\title{
Effects of denatonium saccharide on the drinking behavior of the grasshopper mouse (Onychomys leucogaster)
}

\author{
WILLIAM M. LANGLEY and JOHN THEIS \\ Butler County Community College, El Dorado, Kansas
}

and

\author{
STEPHEN F. DAVIS, M. MELISSA RICHARD, and CATHY A. GROVER \\ Emporia State University, Emporia, Kansas
}

\begin{abstract}
In two experiments, we measured the effects that the bitter denatonium saccharide had on the drinking behavior of the grasshopper mouse. In the first experiment, a 1/1,000 solution of denatonium saccharide, presented every fourth day, suppressed the drinking response. In the two-bottle preference tests of the second experiment, the $1 / 5,000$, but not the $1 / 10,000$, concentration suppressed the mouse's drinking response. These results were similar to those previously reported for the laboratory rat.
\end{abstract}

Denatonium saccharide, a newly discovered chemical, is the most bitter compound known to man ("As Bitter as They Come," 1985). Davis et al. (1986) found that denatonium saccharide did affect the drinking behavior of the albino laboratory rat. More specifically, concentrations of $1 / 1,000$ and $1 / 5,000$ consistently suppressed drinking in a two-bottle preference test, but produced only transient effects in a one-bottle test. In order to determine how widespread denatonium saccharide's aversive effects are, we performed the present experiments to measure the effects that denatonium saccharide had on the drinking behavior of the grasshopper mouse (Onychomys leucogaster). The approach used here was similar to that used by Davis et al. (1986) with the laboratory rats. Experiment 1 measured the effect when a $1 / 1,000$ concentration was presented in a one-bottle test. Because the one-bottle testing procedure creates a restrictive drinking schedule that may force the animal to consume denatonium saccharide when the animal might not otherwise do so, the procedure was changed to a two-bottle preference test for the weaker solutions of $1 / 5,000$ and $1 / 10,000$ employed in Experiment 2.

\section{EXPERIMENT 1}

\section{Method}

Subjects and Apparatus. Five northern grasshopper mice served as subjects. Two were live-trapped in the field, whereas 3 were born and reared in the laboratory. The mice weighed from 30 to $45 \mathrm{~g}$ and were

Appreciation is expressed to Atomergic Chemetals Corporation, 91 Carolyn Blvd., Farmingdale, NY 11735-1527 for supplying the denatonium saccharide. Requests for reprints can be addressed to either Stephen F. Davis, Department of Psychology, Emporia State University, Emporia, KS 66801 , or William M. Langley, Department of Biology, Butler County Community College, El Dorado, KS 67042. at least 70 days old at the time of testing. All animals were individually housed in clear plastic cages that measured $18 \times 28 \times 23 \mathrm{~cm}$. The subjects were maintained in an animal room having a natural daylight cycle. The animals had free access to Purina Laboratory Chow for the duration of the experiment. All tests were conducted in the home cage.

Procedure. Seven days prior to the start of testing, the animals were placed on water deprivation and given access to water for $10 \mathrm{~min}$ at approximately the same time each day. This general procedure continued throughout the testing phase with the mice receiving five cycles of the following 4-day fluid-presentation schedule: distilled water was presented on Days 1-3, and the $1 / 1,000$ denatonium saccharide solution was presented on Day 4 . Thus, testing encompassed a total of 20 days. All fluids were presented in graduated 50-ml centrifuge tubes fitted with spill-resistant sippers. The amount of fluid consumed (in milliliters) by each subject on each test trial was recorded.

\section{Results and Discussion}

Visual inspection of the data showed that the drinking behaviors of the lab-reared and field-caught animals were similar. Therefore, the responses of all 5 mice were pooled. During the last 2 days of the baseline period, mean water consumption was approximately $6 \mathrm{ml}$ per day. As can be seen from Figure 1, this is similar to the consumption rate of the first 3 days of testing. Figure 1 clearly indicates that every fourth day, when the denatonium saccharide was presented, there was a marked decrease in fluid consumption. This graphic impression was corroborated by a significant days effect $[F(19,57)$ $=10.40, p<.001]$. Subsequent Newman-Keuls tests indicated that the subjects consumed significantly $(p<.01)$ less fluid on Days 4, 8, 12, 16, and 20 (i.e., during denatonium saccharide presentations) than on the other days of the test phase. There appeared to be a possible increase in denatonium saccharide consumption by the end of the fourth and fifth cycles (i.e., Days 16 and 20); however, the subjects $\times$ days interaction failed to yield significance $[F(19,57)=1.32, p>.05]$. Clearly, a 


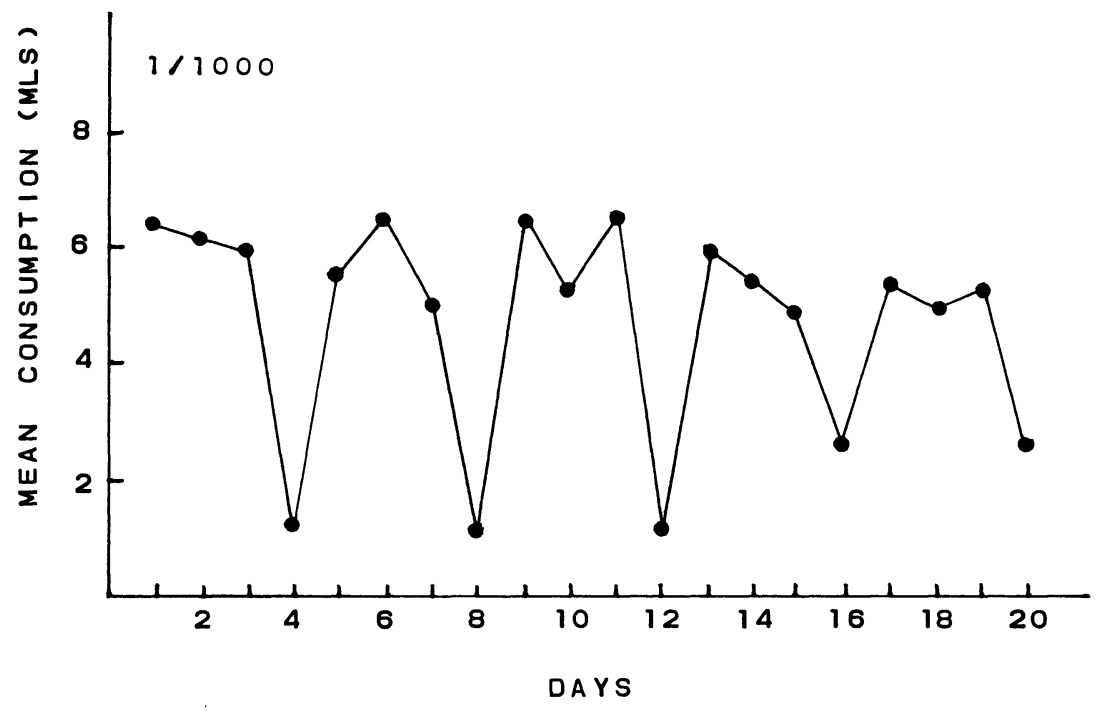

Figure 1. Experiment 1: mean fluid consumption over five cycles of a 3-day water/1-day 1/1,000 denatonium saccharide solution presentation sequence.

$1 / 1,000$ solution of denatonium saccharide inhibited the grasshopper mouse's drinking response, but did not totally stop it.

\section{EXPERIMENT 2}

\section{Method}

Subjects and Apparatus. Ten laboratory-reared, naive grasshopper mice served as the subjects in Experiment 2. The subjects were randomly assigned to two equal groups: $1 / 5,000$ and $1 / 10,000$ denatonium saccharide solutions. Housing and food-maintenance conditions were the same as in Experiment 1. Testing took place in the home cage.

Procedure. During baseline and test sessions, each animal had access to fluid(s) for $10 \mathrm{~min}$. To ensure that each animal had sufficient water each day, these 10 -min sessions were followed $90 \mathrm{~min}$ later by a supplemental 30-min water-access period. The first 7 days of the experiment consisted of the establishment of a water baseline. Testing en- compassed the ensuing 10 days. During testing, each animal was simultaneously presented two 50 -ml centrifuge tubes containing either the assigned denatonium saccharide solution or water. Position of the centrifuge tubes on the cages during testing was randomly assigned daily.

\section{Results and Discussion}

For both groups, the mean fluid consumption during baseline did not differ reliably from the total amount drunk on each day of the test phase (i.e., approximately $6 \mathrm{ml}$ ). Figure 2 indicates that the consumption of the $1 / 5,000$ denatonium solution was substantially less than water consumption for these animals. In support of the graphic data, the fluids factor was found to be significant $[F(1,9)=$ $25.00, p<.01]$.

The performance of the $1 / 10,000$ mice is shown in Figure 3. Even though less denatonium was consumed

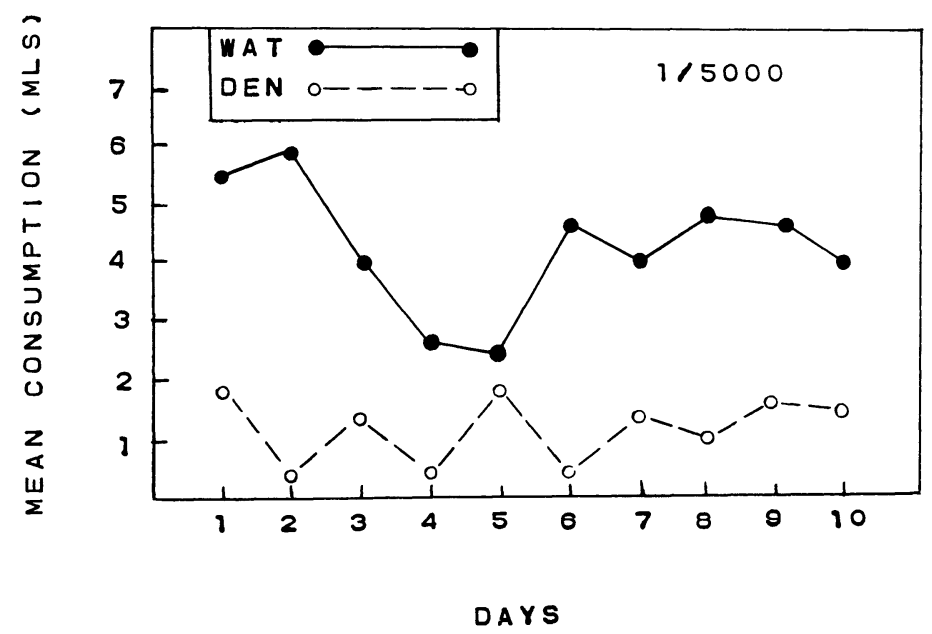

Figure 2. Experiment 2: mean fluid consumption of water and 1/5,000 solution of denatonium saccharide during the 10-day test phase. 


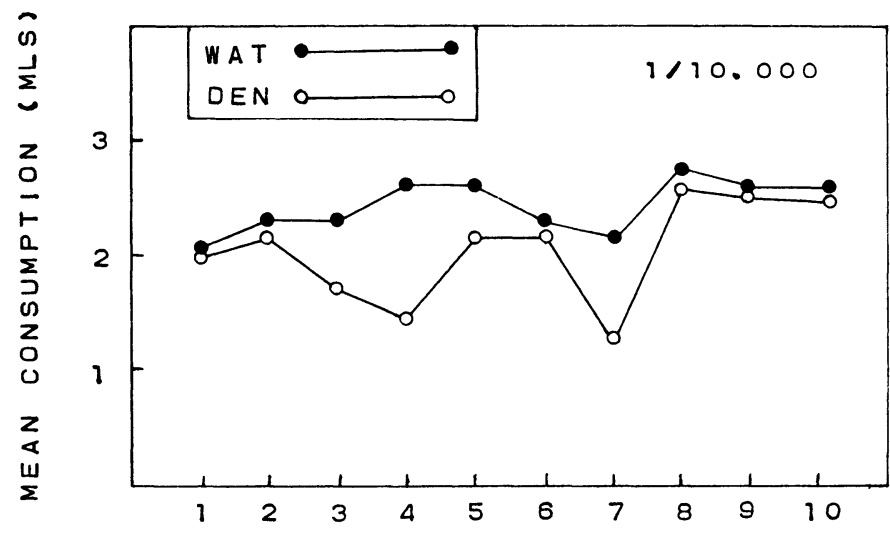

DAYS

Figure 3. Experiment 2: mean fluid consumption of water and 1/10,000 solution of denatonium saccharide during the 10-day test phase.

than was water, this difference was not reliable $[F(1,9)$ $=.97, p>.05]$. In summary, during a two-bottle-choice test, a $1 / 5,000$ but not a $1 / 10,000$ solution of denatonium saccharide significantly suppressed the drinking response of the grasshopper mouse.

\section{GENERAL DISCUSSION}

These data show that denatonium saccharide solutions can suppress the grasshopper mouse's drinking response. Concentrations of $1 / 1,000$ (Experiment 1) and 1/5,000 (Experiment 2) suppress the total volume of fluid drunk, but do not totally inhibit drinking. In a previous report (Davis et al., 1986) we demonstrated the suppression of the drinking response of laboratory rats with the same concentrations of denatonium saccharide. It would appear, however, that the $1 / 1,000$ solution of denatonium saccharide may have suppressed the responses of the grasshopper mouse more completely than those of the rat. The present data (Experiment 1) indicate that suppression was shown on all five denatonium presentations, whereas suppression was shown by the rats only on the first three presentations. Furthermore, this concentration completely suppressed drinking one or two times in 4 grasshopper mice compared with no complete suppressions in the rats. This fact is interesting given that the daily amount of water consumed by a grasshopper mouse represents $16 \%$ of the mouse's body weight. On the other hand, the rat's consumption of water amounts to less than $10 \%$ of its body weight. In summary, it is clear that denatonium saccharide in relatively strong concentrations can suppress, but not completely stop, the drinking response of both grasshopper mice and laboratory rats.

\section{REFERENCES}

As bitter as they come. (1985). Science News, 123, 9.

Davis, S. F., Cunningham, L. A., Burke, T. J., Richard, M. M., LANGLEY, W., \& THEIS, J. (1986). A preliminary analysis of the suppressive effects of denatonium saccharide. Bulletin of the Psychonomic Society, 24, 229-232.

(Manuscript received for publication August 20, 1986.) 